\title{
ESTUDO DE PROBLEMAS DE ENFERMAGEM REFERENTES AO CABELO E COURO CABELUDO EM PACIENTES HOSPITALIZADOS*
}

\section{Maria Romana Friedlander**}

FRIEDLANDER, M. R. - Estudo de problemas de enfermagem referentes a cabelo e couro cabeludo em pacientes hospitalizados. Rev. Esc. Enf. USP, 10(2): 260-273, 1976.

A autora procurou identificar os tipos e os problemas de enfermagem referentes ao cabelo e couro cabeludo, apresentados por pacientes internados em clínicas médicas e cirúrgicas gerais de um hospital governamental de ensino. Identificou 4 classes de problemas: estado de limpeza, lesões, infestações e penteação. Cada classe foi subdividida em graus os quais foram denominados tipos de problemas. A proporção de problemas por paciente encontrada foi 0,80 e a proporção de pacientes com problemas foi 58\%. Foram também estudadas algumas condições que podem intervir no cuidado ao cabelo e ao couro cabeludo e foram obtidas informações sobre hábitos e costumes apresentados pelos pacientes.

\section{INTRODUÇÃO}

O cabelo que recobre o crânio do ser humano desempenha dois tipos de função que poderiam ser denominados funçōes fisiológicas e funções psicológicas. As fisiológicas se referem à proteção contra os extremos de temperatura e ao auxílio prestado à percepção táctil. As funções de ordem psicológica dizem respeito ao papel desempenhado ${ }^{i}$ em nossa sociedade pela aparência física e à formação da auto-imagem.

Sendo a enfermagem uma profissão que assiste o ser humano, compreende-se que o cuidado ao cabelo e couro cabeludo tenha sido,

\footnotetext{
* Resumo da Dissertação de Mestrado apresentada à Escola de Enfermagem da Universidade de São Paulo em dezembro de 1975.

** Professor Assistente da disciplina Fundamentos de Enfermagem da EEUSP.
} 
sistematicamente, ensinado na disciplina Fundamentos de Enfermagem. Entretanto, o conteúdo das aulas sobre esse assunto tem-nos parecido ser, em geral, baseado nos textos dos livros básicos de Enfermagem e pouca ou nenhuma contribuição tem sido acrescida nos últimos anos.

O presente trabalho tem as seguintes finalidades: a) identificar os tipos e 0 número de problemas de enfermagem referentes a cabelo e couro cabeludo, apresentados em pacientes hospitalizados, adultos e internados em clínicas médicas e cirúrgicas de um hospital governamental de ensino; b) estimar a proporção de pacientes que apresentem esses problemas de enfermagem; c) verificar uma possível relação entre a existência de pacientes com problemas e algumas características da população estudada; d) estudar algumas condições que possam intervir no cuidado ao cabelo e couro cabeludo; e) obter informações sobre condições e hábitos apresentados pelos pacientes que possam servir de subsídio para o planejamento de cuidados.

Os livros-texto básicos de Enfermagem trazem referências ao cuidado com o cabelo e couro cabeludo, salientando os princípios que o regem e a sua importância para o bem estar e conforto do paciente (HARMER \& HENDERSON, 1959; FUERST \& WOLFF, 1964; PRICE, 1966; McCLAIN \& GRAGG, 1970; DuGAS, 1972; MATHENEY et al, 1972; MITCHELL, 1973).

Contudo, na literatura consultada foram encontrados poucos estudos referentes a esses problemas de enfermagem. HORTA (1968) aplicando um método de observação sistematizada encontrou, em 46 pacientes, 22 problemas relacionados ao cabelo e couro cabeludo, equivalentes a 0,48 problemas por paciente. PORTO (1969) em um estudo feito em pacientes acamados de um hospital do Rio de Janeiro, analisa os componentes de higiene e conforto físico para a avaliação do cuidado de enfermagem: dos 33 pacientes estudados encontrou $8(24,24 \%)$ com o couro cabeludo e cabelos limpos. VIEIRA et al (1971) fizeram um levantamento do conteúdo das anotações de enfermagem. No ítem referente à higiene da cabeça encontraram $152(97,5 \%)$ anotações computadas como "inexistentes". $\mathrm{O}$ termo inexistente foi utilizado para classificar as anotações ou observações requeridas pelo estado do paciente mas não encontradas no prontuário ou kardex.

Para conceituarmos o problema de enfermagem referente a cabelo e couro cabeludo, analisamos diversos livros-texto básicos de enfer- 
magem. Cada autor indica as atividades que caracterizam as funções da enfermagem nesta área. Deduzimos que o conjunto dessas atividades delimita a responsabilidade da profissão no que concerne à solução dos problemas específicos da área. Consideramos, assim, a penteação, a escovação, a lavagem da cabeça e a prevenção e o tratamento da pediculose craneana, como cuidados pelos quais a enfermagem é responsável. E responsável ainda pela prevenção de lesões no couro cabeludo, pela promoção da sua integridade e pelo auxílio na reabilitação dessa integridade quando ela estiver lesada.

Para fins deste trabalho, consideramos como problemas de enfermagem referentes a cabelo e couro cabeludo as seguintes condições: cabelo despenteado, cabelo emaranhado, cabelo e/ou couro cabeludo sujos, pediculose craneana e lesões do couro cabeludo.

\section{METODOLOGIA}

O estudo foi feito no Hospital das Clínicas da Faculdade de Medicina da Universidade de São Paulo. Para o levantamento dos dados utilizou-se uma amostra de 100 indivíduos de uma população constituída por pacientes com as seguintes características: conscientes, com idade superior a 12 anos, internados em uma das clínicas médicas e cirúrgicas gerais do referido hospital, que não estivessem recebendo cuidados em unidades de terápia intensiva, salas de choque, salas de recuperação pós-operatória, salas de diálise e salas de recuperação cardíaca.

Para a coleta de dados foi utilizado um formulário (Anexo) preenchido pela própria autora. Para a aplicação do formulário foi mantido o horário fixo das 9 horas e 30 minutos às 11 horas e 30 minutos com ạs seguintes finalidades: a) não coincidir com a visita médica; b) não coincidir com horário de refeições; c) não coincidir com horários de visitas; d) evitar as altas e admissões durante a seleção dos pacientes e a aplicação do formulário; e e) dar oportunidade para que todos os pacientes estivessem nas mesmas condições de higiene.

A técnica usada para a aplicação do formulário foi a seguinte:

$1 .^{\circ}$ passo - Estudo da ficha-leito do paciente: levantados os dados de identificação e características gerais do paciente, confirmadas, posteriormente, durante a entrevista. 
2. ${ }^{\circ}$ passo - Observação direta: Material utilizado - bolas de algodão embebidas em éter, papel absorvente e uma pequena régua milimetrada; técnica - com o algodão esfregamos o couro cabeludo verificando-se o "grau" de pó; em seguida, passamos o papel absorvente para a identificação da oleosidade e com a régua milimetrada medimos uma ondulação do cabelo classificando o seu tipo. Nessa etapa observamos, ainda, a existência de descamação e a sua graduação, o aparecimento e tipos de lesão, infestações, odor, fạlta de cabelo, comprimento, material encontrado no criado-mudo e uso de peruca.

3. passo - Entrevista com o paciente: coletados os demais dados e confirmados os dados da ficha-leito.

Antes da aplicação do formulário, estabelecemos todos os critérios necessários e elaboramos as linhas gerais da classificação dos problemas, cujo resumo apresentamos:

EST ADO DE LIMPEZA - avaliado pela soma dos "graus" de pó, de oleosidade e de descamação, soma esta que varia de zero a seis. Os graus de limpeza " 0 " e " 1 " definem o estado de limpo e, portanto, não constituem problema de enfermagem. Os graus " 2 " e " 3 " definem um estado em que a cabeça deve ser lavada sem ser, ainda, um cuidado prioritário. Os graus " 4 ", " 5 " e " 6 " definem o estado em que a cabeça deve ser lavada o mais rápido possível.

LESOES - Foram classificadas em 3 graus. O grau "0" foi definido quando não existia lesões; o grau " 1 " foi computado quando a região da cabeça ocupada pelas lesões não ultrapassava cinco centímetros de diâmetro; e o grau " 2 " quando a região ocupada pelas lesões ultrapassava cinco centímetros de diâmetro.

INFEST AÇOES - O grau " 1 " foi definido quando verificamos a existência de lêndeas sem o aparecimento visível do inseto; e o grau "2" quando verificamos o aparecimento do Pediculus $h$. humanus.

PENTEAÇÃO - O grau "1", ou despenteado, foi computado quando verificamos a existência de mechas de cabelo não ordenadas (excluindo-se cabelos eriçados com finalidade estética); e grau " 2 " quando foram encontrados nós visíveis de cabelo emaranhado. 
Ainda caracterizamos e classificamos as condições que denominamos sintomas que poderão indicar a existência de um problema e que são: odor desagradável, prurido, queda de cabelos e dor no couro cabeludo. Foi feito o mesmo com as condições que podem intervir no cuidado ao cabelo e couro cabeludo: calvície e alopécia, comprimento e tipo de cabelo, existência de pente, escova, sabonete, xampu, cosméticos e peruca, e cuidados que está recebendo no hospital.

Optamos pela análise dos dados através de tabelas de contingência e pela utilização do Teste do Qui-Quadrado (SIEGEL, 1956; e RODRIGUES, 1975).

\section{ANÁLISE E DISCUSSÃO DOS RESULTADOS}

a) Características gerais da população estudada

Entre os 100 pacientes estudados, apresentaram maioria absoluta (mais do que $50 \%$ ) os:

- do sexo feminino - 52\%;

- adultos (18 - 60 anos) $-77 \%$;

- católicos - 86\%;

- brasileiros - $94 \%$;

- procedentes de outras localidades que não fosse $\mathrm{o}$ município de São Paulo - 54\%;

- com diagnóstico médico-clínico - 53\%;

- ambulantes - $84 \%$.

Ainda se salientaram os seguintes resultados: estado civil: $45 \%$ dos pacientes eram casados e $45 \%$ solteiros; ocupação: $45 \%$ dos pacientes possuiam ocupações que não exigem especialização alguma, tais como prendas domésticas, faxineiro, operário não especializado, vendedor ambulante, e outras; instrução: $49 \%$ dos pacientes eram analfabetos ou semi-alfabetizados e $40 \%$ tinham instrução primária completa; dias 
de internação: $41 \%$ dos pacientes estavam internados entre 01 e 10 dias e $32 \%$ entre 11 e 30 dias.

b) Distribuição e classificação dos problemas encontrados tes denominações:

Para a apresentação desta parte convencionamos as seguin-

- classes de problemas - quando nos referimos ao estado de limpeza, às lesōes, às infestações e à penteação;

- tipos de problemas - são os graus em que estão subdivididas as classes. Contudo, os graus " 0 " e " 1 " do estado de limpeza e o grau " 0 " das demais classes não constituem problema e, portanto, são excluídas da denominação "tipos de problemas".

Foram encontrados problemas referentes às quatro classes definidas, como se pode verificar na Tabela $\mathrm{I}$.

TABELA I

DISTRIBUIÇÃO PORCENTUAL DE PACIENTES SEGUNDO A CLASSE E OS TIPOS DE PROBLEMAS

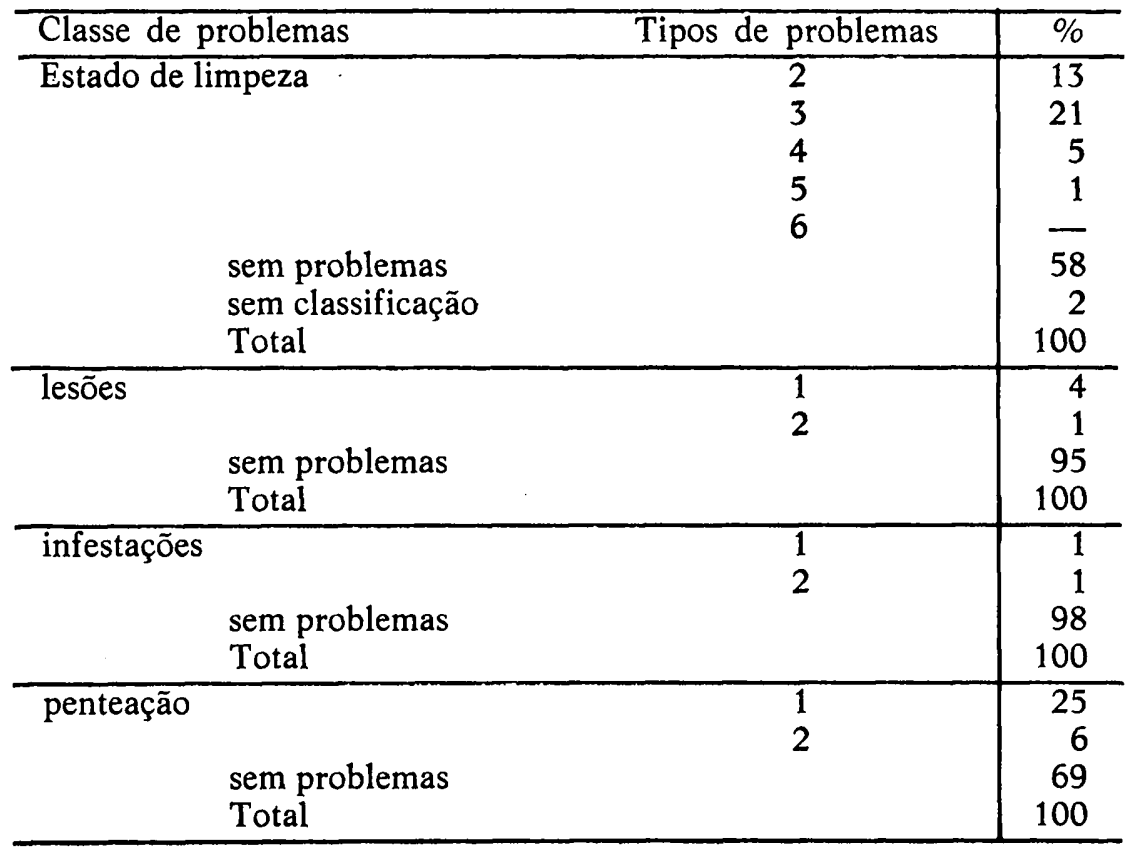


PORTO (1969) encontrou somente $24,24 \%$ dos pacientes com o cabelo e couro cabeludo limpos, enquanto nós encontramos $58 \%$. A diferença de porcentagem deve-se, talvez, ao fato daquele autor ter estudado pacientes acamados e, possivelmente, utilizado critérios diferentes. Do total, $6 \%$ dos pacientes apresentaram problemas referentes ao estado de limpeza nos graus " 4 " e " 5 ", e não foi encontrado paciente algum com o grau "6" de limpeza. HORTA (1968) também não encontrou pacientes no estado de limpeza em grau "péssimo" de sua classificação.

As porcentagens referentes às lesões e infestações foram baixas mas são classes de problemas que exigem da enfermagem, para sua solução, conhecimentos específicos e não podem ser deixados de lado.

O tipo de problema que apareceu em maior porcentagem de pacientes foi o grau " 1 " de penteação $(25 \%)$. Esse número pode ser explicado pelo fato de que os pacientes, mesmo ambulantes, utilizem o leito para quase todas as suas atividades.

Tentamos verificar a existência ou não de uma associação entre as diferentes classes de problemas. Alguns resultados merecem descrição. Todos os pacientes que apresentaram lesões também apresentaram problemas de limpeza, e todos os pacientes com pediculose apresentaram, simultaneamente, problemas de limpeza. Entretanto, verificamos uma independência estatística entre o número de pacientes com problemas de limpeza e de penteação.

Como cada paciente teve a possibilidade de apresentar, simultaneamente, de zero a quatro problemas, apresentamos a Tabela II.

TABELA II

DISTRIBUIÇÃO DOS PACIENTES SEGUNDO O NƯMERO DE PROBLEMAS ENCONTRADOS

\begin{tabular}{c|c|c}
\hline $\begin{array}{c}\text { Número de problemas } \\
\text { por paciente }\end{array}$ & $\begin{array}{c}\text { Número de } \\
\text { pacientes }\end{array}$ & $\begin{array}{c}\text { Total de } \\
\text { problemas }\end{array}$ \\
\hline 0 & 43 & 0 \\
1 & 36 & 36 \\
2 & 15 & 30 \\
3 & 4 & 12 \\
4 & 0 & 0 \\
sem classificação & 2 & - \\
\hline TOTAL & 100 & 78 \\
\hline
\end{tabular}


A média de problemas por paciente é 0,80 , ou seja, a enfermagem pode prever que, em cada 100 pacientes hospitalizados, poderão ser encontrados 80 problemas. HORTA (1968) encontrou uma média de 0,48 problemas por paciente. A diferença entre as médias possivelmente se deve à diferença entre os critérios utilizados, a época de realização dos trabalhos e as condições do levantamento dos dados.

A proporção de pacientes com problemas encontrada é igual a $56,12 \%$.

Quanto aos resultados sobre os sintomas que poderão indicar a existência de algum problema, mencionamos os que mais se destacam. O prurido foi mencionado por $14 \%$ dos pacientes, a queda de cabelos por $25 \%$, o odor desagradável verificado em $5 \%$ de pacientes, e a dor não foi referida por paciente algum. Encontramos uma associação estatisticamente significante entre os problemas de limpeza e o prurido. Contudo, verificamos que, dos $\mathbf{4 0}$ pacientes com problemas de limpeza, somente 10 mencionaram o prurido. Dos 5 pacientes que apresentaram odor desagradável, 4 também apresentaram problemas de limpeza. $O$ estudo sobre a possível relação entre a queda de cabelos e problemas de limpeza mostrou uma independência estatística entre esses dois fatores. $O$ estudo das possíveis relações restantes foi prejudicado devido ao pequeno número de pacientes com infestações e lesões.

Esses sintomas mereceriam ser objeto de outros estudos.

c) Relação entre as características gerais da população e o número de pacientes com problemas

Nesse sentido também houve, de nossa parte, uma preocupação em analisar, estatisticamente, cada uma das características gerais da população e o número de pacientes com problemas. $O$ estado de acamado influiu significantemente para o aparecimento de maior número de pacientes com problemas. Podiamos esperar essa influência, levando em consideração que:

- as condições de higiene no leito são mais precárias do que nas instalaçōes sanitárias; 
- os pacientes acamados são mais dependentes da enfermagem que, nem sempre tem condições de prestar, no leito, um cuidado de higiene de alto nível;

- muitos dos pacientes acamados apresentam outras espécies de problemas de tal importância para a manutenção da vida que os de higiene se tornam secundários $\mathrm{e}$

- os pacientes acamados têm maior número de limitações físicas que os impedem de manter um alto nível de higiene corporal.

A análise da relação entre sexo, estado civil, procedência, nível de instrução, diagnóstico médico e a existência ou não de problemas levou-nos a aceitar o fato de que essas características não aumentam as possibilidades de aparecerem mais ou menos pacientes com problemas. Não houve condiçōes numéricas para analisarmos a possível existência de uma relação entre idade, nível de ocupação e um maior ou menor número de pacientes com problemas.

d) Hábitos do paciente e condições que podem intervir no cuidado ao cabelo e couro cabeludo.

Este ítem refere-se especificamente aos dois últimos objetivos deste estudo. Os nossos resultados demonstraram que a falta, o comprimento e o tipo de cabelo não influem no número de pacientes com problemas. Verificamos também que somente a posse de pente ou escova e de sabonete ou xampu não interfere para diminuir ou aumentar os problemas de limpeza. Na nossa prática diária temos observado que existem outros fatores (tais como conhecimento e hábitos de higiene e condições físicas ou ambientais) que determinam uma boa lavagem da cabeça. Acrescentamos ainda que o estado de conservação do material encontrado no criado-mudo deve merecer a atenção da enfermagem, no aspecto de orientação e educação sanitária. Encontramos uma associação positiva significante entre o número de dias transcorridos desde a última vez que o paciente lavou ou penteou o cabelo e o número de problemas de limpeza e penteação, respectivamente.

Foram obtidas dos pacientes algumas sugestões quanto aos cuidados que gostariam lhes tivessem sido prestado. Destaca-se "cortar o cabelo", sugestão apresentada por 8 pacientes. Esta e outras sugestões 
apresentadas levam-nos a aceitar e a recomendar a idéia de que os hospitais tenham um barbeiro ou cabelereiro à disposição dos pacientes. Ainda indicam que cada paciente tem necessidades individuais $e$, como tais devem ser atendidas.

Levantaram-se alguns costumes e hábitos dos pacientes estudados. Vinte e um pacientes informaram não lavar a cabeça quando estão doentes (principalmente com febre). Os motivos religiosos apareceram como proibitivos do corte de cabelo em mulher e da manutenção do cabelo longo em homens. Os períodos menstrual e puerperal também apareceram como fatores que impedem algumas mulheres de lavarem a cabeça.

Se a enfermagem é uma profissão eminentemente de atendimento ao indivíduo e se cada ser humano deve participar ativamente de seus cuidados, o conhecimento de seus hábitos torna-se de interesse para a prática da enfermagem. Isto não significa que os possamos generalizar para todos os pacientes. Pelo contrário, deve-se dar maior ênfase para que a enfermagem procure levantar os hábitos de cada um de seus pacientes, ao invés de sistematizar rotineiramente seus cuidados. Assim, tomar conhecimento dos costumes mais comuns poderá auxiliar a prever os dados que deverão ser levantados de cada paciente e contribuir para o planejamento da assistência de enfermagem.

\section{CONCLUSOES}

Os objetivos do estudo foram plenamente atingidos. Desse trabalho podemos tirar as seguintes conclusões gerais:

- foram identificados problemas de enfermagem referentes ao estado de limpeza, infestações, lesões e penteação; salientaram-se os tipos estado de limpeza graus " 2 " e " 3 " e penteação grau " 1 ";

- com os critérios utilizados pudemos atingir um nível razoável de objetividade na classificação dos problemas; esses critérios poderão ser usados na prática de enfermagem para avaliar o estado do cabelo e couro cabeludo do paciente hospitalizado;

- os sintomas prurido, queda de cabelos e odor desagradável não parecem ser específicos de uma classe de problemas: são indícios gerais de que existem problemas inespecíficos; 
- o material usado pelo paciente para seus cuidados de higiene corporal também deve ser objeto de cuidados por parte da enfermagem;

- cada indivíduo tem hábitos e costumes referentes à higiene da cabeça que devem ser do conhecimento da enfermagem e considerados no planejamento de cuidados;

- outros estudos deveriam ser realizados, principalmente, no que se refere a:

- sintomas dos problemas de enfermagem referentes a cabelo e couro cabeludo,

- influência de moléstias endocrinológicas e dermatológicas no aparecimento dessa espécie de problemas,

- importância do cabelo sob o ponto de vista do paciente,

- problemas de cabelo e couro cabeludo em pacientes com dependência total de enfermagem, tipo de problemas.

- cuidados de enfermagem específicos para cada classe ou

FRIEDLANDER, M. R. - Study about nursing problems of hospitalized patients related to hair and scalp. Rev. Esc. Enf. USP, 10(2): 260273, 1976.

The author tried to identify types and number of hair and scalp nursing problems presented by medical-surgical patients of a governmental teaching hospital. Four (4) classes of problems were identified: cleanliness conditions, lesions, infestation and combing conditions. Each class was sub-divided into a scale, named types of problems. The proportion of detected problems, per patient, was of 0,80 and the proportion of patients with problems was of $58,00 \%$. Some conditions that may interfere in hair and scalp care were also studied and some information gathered on habits and practices of subjects. 


\section{REFERENCIAS BIBLIOGRÁFICAS}

DuGAS, B. W. (KOZIER, B. B.) - Introduction to patient care. 2nd. ed. Philadelphia, W. B. Saunders. 1972. p. 145.

FUERST, E. V. \& WOLFF, L. V. - Fundamentals of nursing. 3rd ed. Philadelphia, Lippincott. 1964. p. 297.

HARMER, B. \& HENDERSON, V. - Tratado de enfermeria teorica y practica. 2ed. trad. Mexico, La Prensa Medica Mexicana. 1959. p. 380.

HORTA, W. de A. - A observação sistematizada na identificação dos problemas de enfermagem em seus aspectos físicos. São Paulo, Serviço de Documentação da RUSP. 1968, p. 8 e 20. (Tese de Livre Docência - Escola de Enfermagem da USP).

MATHENEY, R. et al. - Fundamentals of patient-centered nursing. 3rd. ed. Saint Louis, Mosby. 1972. p. 117.

McClAIN, \& GRAGG, S. H. - Principios científicos de enfermagem. 2ed. Rio de Janeiro, Científica. 1970. p. 217.

MITCHELL, P. H. - Concepts basic of nursing. New York, Mc GrawHill. 1973. p. 415 e 419.

PORTO, M. E. A. - Análise do nível de assistência de enfermagem. Servir, 1:138, ago/nov. 1969.

PRICE, A. L. - Tratado de enfermeria. 3 ed. Mexico, Interamericana. 1966. p. 29 e 127.

RODRIGUES, A. - A pesquisa experimental em psicologia e educação. Rio de Janeiro, Vozes. 1975. p. 195 e 207.

SIEGEL, S. - Nonparametric statistics for the behavioral sciences. Tokyo, International Student Edition, McGraw-Hill and Kogakusha Company, 1956.

VIEIRA, A. et al. - O princípio da investigação e observação sistematizada em enfermagem: uma experiência em hospital-escola. Rev. Bras. Enf., 24(5): 66-89, jul/set. 1971. 


\begin{abstract}
ANEXO
Formulário N. ${ }^{\circ}$

Data de Preenchimento:

Iniciais do Pt.:

Reg.:

Clínica:

Sexo:

Idade:

Religião:

Nacion.:

Proced.:

Est. Civil:

Ocupação:

Instrução:

Data de Adm.:

Diagnóstico Médico:

Deambulação: Acam.:

Em rep. relativo:

Ambul.:
\end{abstract}

\title{
PROBLEMAS DE C̣ABELO E COURO CABELUDO
}

Estado de Limpeza: Impressão Pessoal:

Pó n/existente (0): . . . presente (1): . . . acumulado (2):

Oleosidade: normal (0): . . oleoso (1): . . muito oleoso (2): . .

Descamação: nula (0): $\ldots$ pequena (1): $\ldots$ acentuada (2): $\ldots$

Lesões $\mathrm{n} /$ existentes $(0)$ :

existentes:
localiz. (1): $\ldots \ldots$
general. (2): $\ldots \ldots$

Infestações: $n$ /existente $(0): \ldots$ lendeas (1): $\ldots$ pediculose (2):

Penteação: penteado (0): $\ldots$ despenteado (1): . . emaranhado (2):

Odor: ausente: $\ldots \ldots \ldots$ agradável: $\ldots \ldots \ldots$ n/agradável:

Queixas do Pt.: prurido: .... . dor: ... . . queda ocas. de cab.: outras:

\section{CONDIÇOEES QUE PODEM INTERVIR NOS CUIDADOS AO CABELO E COURO CABBELUDO}

Queda de cabelo: calvície alopécia:

Comprimento: curto: médio: Regiōes:

Tipo de cabelo: liso: ondulado: longo:

Material encontrado no criado-mudo:

Pente: $\ldots$ limpo: $\ldots$ sujo: $\ldots$ quebrado: $\ldots$ íntegro: $\ldots$ Escova: ... limpa: .. suja: .. quebrada: .. íntegra: ... Sabonete: ... Xampu: ... Cosméticos: ... Outros: 
Peruca: ... limpa: ... suja: ... penteada: ... despenteada:

Por que usa?

Cuidados que está Quem presta Frequiência Data da últirecebendo no Hosp. os cuidados dos cuidados ma vez

Lavar:

Pentear:

Cortar:

Outros:

Sugestões para outros cuidados a serem prestados no Hospital:

Cuidados que o paciente, habitualmente não presta ao cabelo na doença:

Lavar: Cortar:

Outros:

Por que?

Cuidados que a religião não permite que sejam prestados ao cabelo:

Lavar: Cortar:

Outros:

Por que? (para o sexo feminino)

Cuidados que não são prestados, habitualmente, no período menstrual: Lavar: Cortar:

Outros:

Por que?

(para as pacientes com filhos)

Cuidados que não são prestados ao cabelo, habitualmente, durante a gravidez:

Lavar: ........ Cortar:

Outros:

Por que?

Cuidados que não são prestados ao cabelo durante o período puerperal:

Lavar:

Cortar:

Outros:

Por que?

OUTRAS OBSERVAÇOESS OCASIONAIS: 